\title{
Acknowledgement to Reviewers of Philosophies in 2020
}

\author{
Philosophies Editorial Office
}

Citation: Philosophies Editorial

Office. Acknowledgement to

Reviewers of Philosophies in 2020.

Philosophies 2021, 6, 8. https://

doi.org/10.3390/philosophies6010008

Published: 26 January 2021

Publisher's Note: MDPI stays neutral with regard to jurisdictional claims in published maps and institutional affiliations.

Copyright: (c) 2021 by the author. Licensee MDPI, Basel, Switzerland. This article is an open access article distributed under the terms and conditions of the Creative Commons Attribution (CC BY) license (http://creativecommons.org/licenses /by/4.0/).

MDPI AG, St. Alban-Anlage 66, 4052 Basel, Switzerland

The editorial team greatly appreciates the reviewers who have dedicated their considerable time and expertise to the journal's rigorous editorial process over the past 12 months, regardless of whether the papers are finally published or not. In 2020, a total of 45 papers were published in the journal, with a median time to first decision of 22 days and a median time from submission to publication of 54 days. The editors would like to express their sincere gratitude to the following reviewers for their generous contribution in 2020:

Arnason, Gardar

Arrigoni, Gabi

Atienza, Elena

Ballesteros, Alfonso

Barfield, Woodrow

Baroni, Bassam El

Basti, Gianfranco

Baumann, Peter

Boltuc, Peter

Bradie, Michael

Brenner, Joseph E.

Canavan, Gerry

Casetta, Elena

Ćirić, Josip

Collins, Sarah

David, Nuno

Davies, Jim

Davis, Benjamin

Davis, Dena

De Lora, Pablo

Dellantonio, Sara

Demski, Abram

Diebschlag, Natalie

Dinneen, Jesse David

Dubljević, Veljko

Edgar, Andrew

English, Colleen

Faye, Jan

Ferrell, Jason

Fields, Chris

Fontaine, Matthieu

Georgescu, Laura

Gere, Charlie

Gimbel, Steve
Grohmann, Kleanthes

Gunji, Yukio-Pegio

Harrigan, James R.

Hermens, Ronnie

Hill, Robin

Kaltsas, Spyridon

Kirchoff, Bruce

Kosta, Peter

Krzanowski, Roman

Lara, Francisco

Lewandowski, Joseph D.

Lister, Andrew

Longo, Giuseppe O.

Loughlin, Michael

Magnani, Lorenzo

March-Russell, Paul

Marijuán, Pedro

Martens, Betsy

Martínez-Ávila, Daniel

Maximova, Marina

McFarlane, Anna

Medley, Stuart

Mende, Doreen

Minkov, Svetozar

Moczek, Armin

Molhoek, Braden

Moon, Kyumin

Nieves, Javier

Norton, John

Oramus, Dominika

Ottarsdottir, Unnur

Paillusson, Fabien

Palmer, Clive

Pellegrino, Emilio 
Persson, Erik

Petros, Stefaneas S.

Pfleegor, Adam G.

Piccinin, Antonella

Pierce, Sarah

Prentner, Robert

Prior, Karen

Repetti, Rick

Reydon, Thomas

Rose, David

Sansom, Dennis

Schreiber, Darren

Schultz, David

Shabo, Seth

Shevlin, Henry
Sholl, Jonathan

Snoek, Anke

Tsukaya, Hirokazu

Turcan, Nicolae

Van Hilvoorde, Ivo

Verdicchio, Mario

W. Carlson, Kristen

Winther, Rasmus

Wiseman, Harris

Wolf, Thomas

Yen, Shi-Jim

Zennaro, Fabio Massimo

Zimmermann, Rainer E.

Zougris, Konstantinos

Żuradzki, Tomasz 